\title{
أهمية دوافع تعلم اللغة العربية لدى الطالبات على همة الإتصال بما \\ (دراسة حالة في برنامج اللغة بمعهد تبوئيرنج الإسلامي للبنات جومبانج)
}

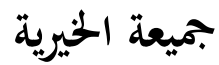

\section{jamiatulkhoiriyah27@gmail.com}

\author{
عبد القدير، الماجستير
}

Tanggal Submitt: 30/9/2019

$$
\begin{aligned}
& \text { الخلاصة :يهفز هذا البحث الدوافع المنخفض لتعلم اللغة العربية في البرامج اللغوية بحيث يقل اهتمامها بالتواصل باستخدام } \\
& \text { اللغة بينما يوجد في البرنامج لغات أخرى ، وهي الإنجليزية. }
\end{aligned}
$$

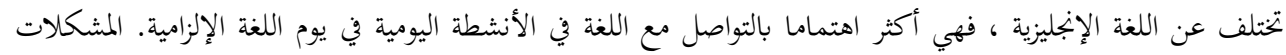

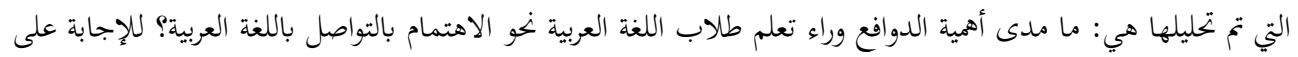

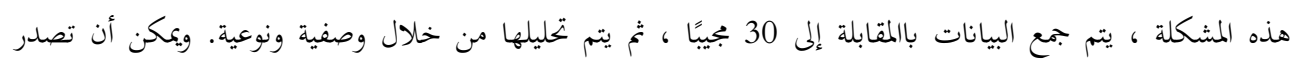

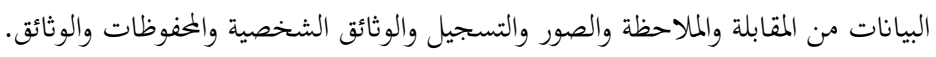

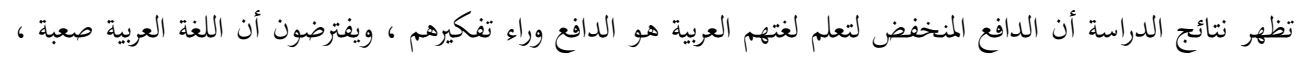

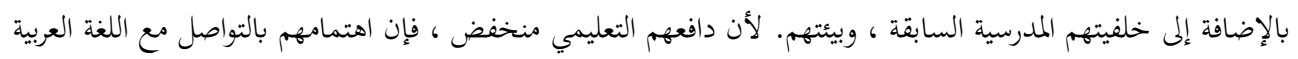

$$
\begin{aligned}
& \text { منخفض أيضا. } \\
& \text { الكلمات المفتاحية: دوافع تعلم، همة، الإتصال. }
\end{aligned}
$$

\begin{abstract}
This research stimulates the low motivation to learn Arabic in the language programs so that they are less interested in communication using the language while the program has other languages,namelyEnglish. Different from English, they are more interested in communicating with the language in everyday activities on mandatory language day. The problems that have been analyzed are: How important are the motivations behind learning Arabic students to be interested in communicating in Arabic? To answer this problem, data is collected by interview to 30 respondents, then analyzed by descriptive and qualitative. The data may be issued from the interview, observation, photographs, registration, personal documents, archives anddocuments. The results of the study show that the low motivation to learn their Arabic language is the motivation behind their thinking, and assume that the Arabic language is difficult, in addition to their previous school background and environment. Because their motivation is low, their interest in communicating with Arabic is also low.
\end{abstract}

Keywords: Motivation To Learn, Passion, Communication. 


\section{مقدمة}

إن اللغة العربية تحتوي من واحدة اللغات العالمية. مع أها كالمواد الإجبارية في المدرسة العالية الإسلامية

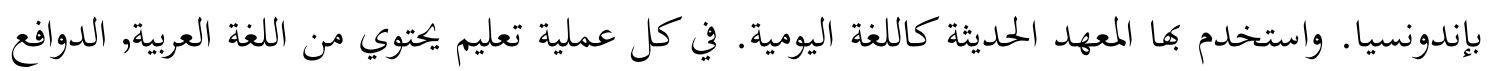

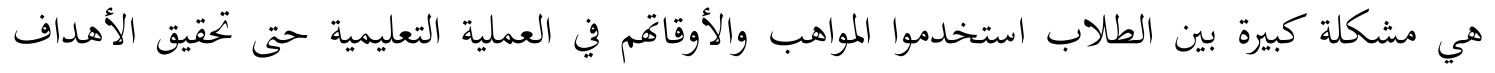
التعليمية تماما. سعى الطلاب ان يستخدموا إختمالمم الذي يزداد عاجلا مع تطوير المواهب لديهم

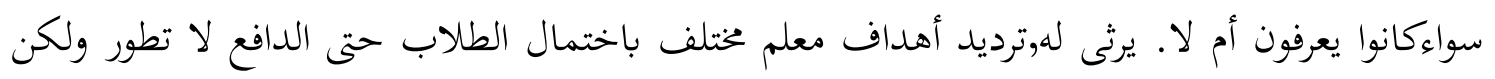

في عملية التعلم، يكون الدوافع ضروريا جدا، لأن شخصا ليس لديه دوافع في التعلم لن يكون من

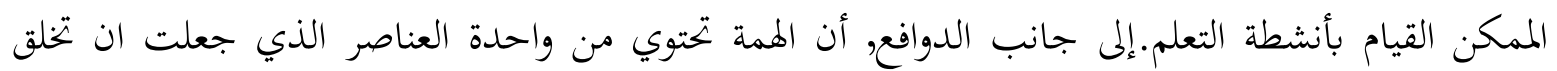
أهداف التعليم، الدوافع محركات وظيفية ونفسية داخل الكائن الحي، تدفعه إلى العمل في الجحاه معين، فتحرك

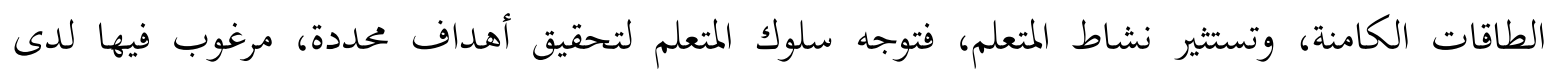

ولكي تنجح عملية التعلم لابد من إثارة هذه الدوافع، ويمكن تحقيق ذلك بإشعار المتعلم بحاجته إلى تعلم

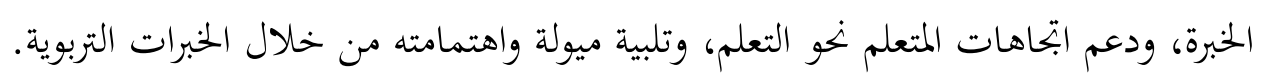

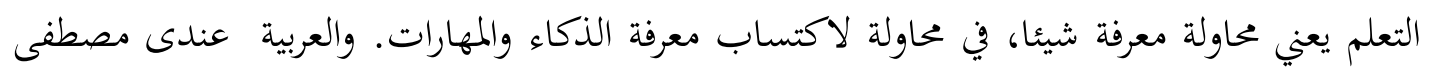

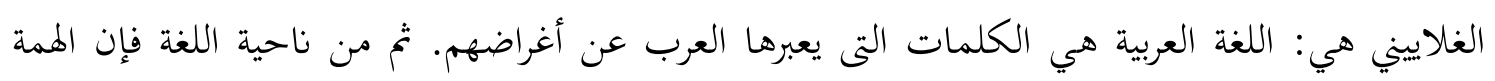

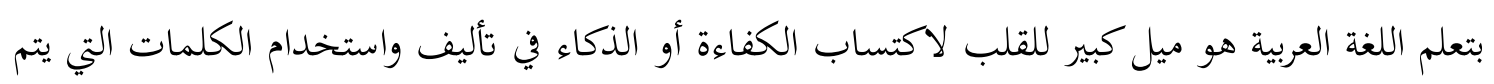

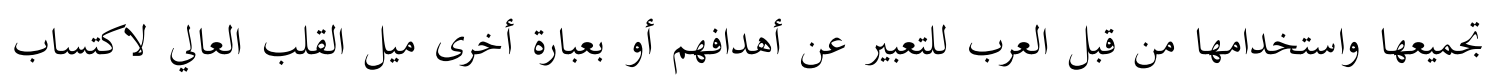

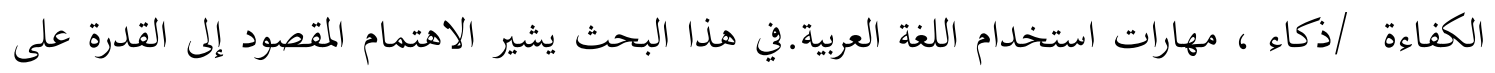

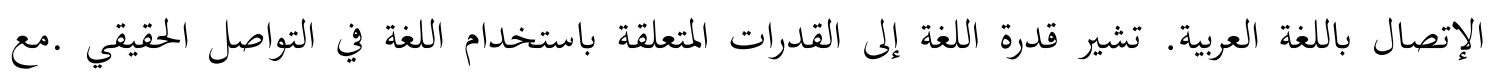

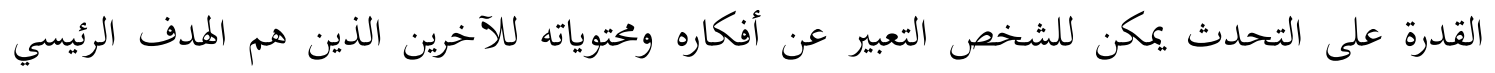

$$
\text { لتدريس اللغة كشكل من أشكال التواصل. }
$$

تأتي الباحثة دراسة حالة حول برنامج اللغة في المعهد تبوئيرنج الإسلامي للبنات جومبانج. في برنامج

$$
\text { اللغة هناك لغتان المحور الرئيسي، وهما اللغة العربية واللغة الإنجليزية، }
$$

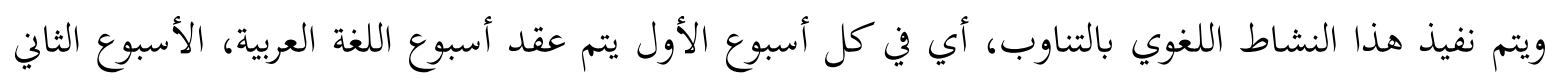

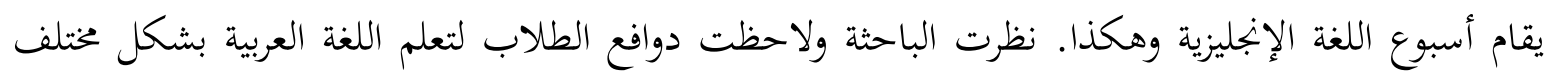


عن دوافعهم لتعلم اللغة الإنجليزية، حين أنه في الوقت برنامج اللغة الإنجليزية نظرا لتردد بين اللغتين الإنجليزية

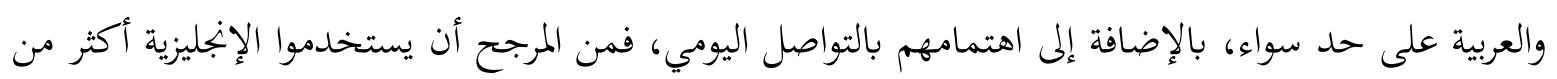
العربية.

لذلك تمتم الباحثة بمناقشة هذه القضية بإعطاء الموضوع " أهمية دوافع تعلم اللغة العربية لدى

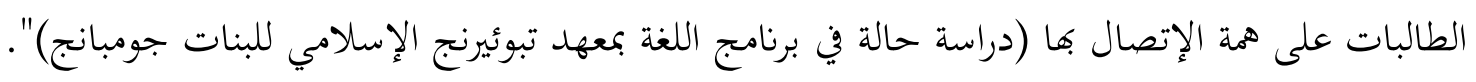

\section{منهج البحث أ. مدخل البحث ونوعه}

في هذا البحث تستخدم الباحثة بالبحث الكيفى الميدانى. البحث الكيفي هو البحث البحث

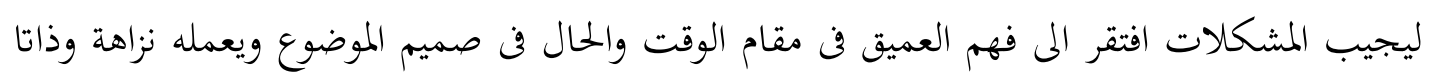

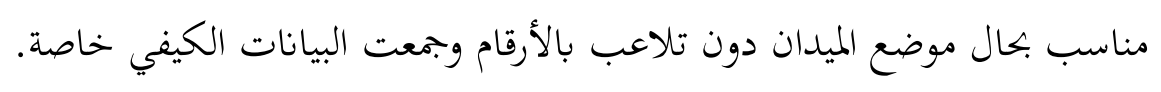

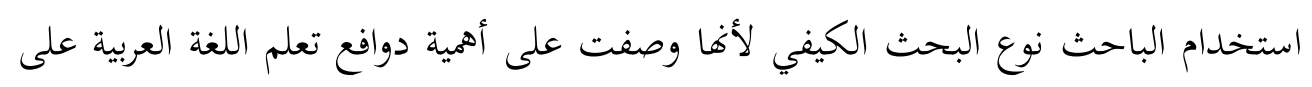

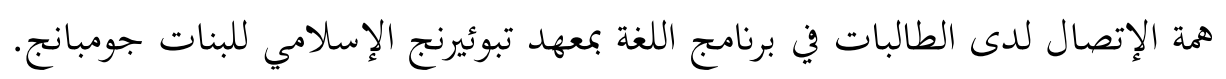

\section{ب. بأدوات البحث}

كانت الباحثة أداة رئيسية في هذا البحث لتعيين المخبرات كمصادر البيانات وجمع البيانات

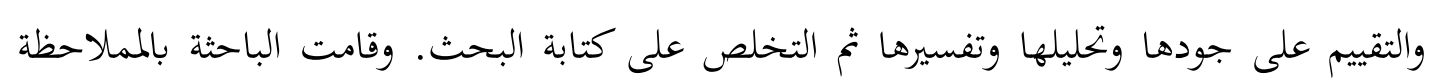

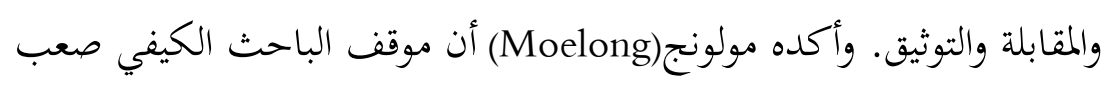

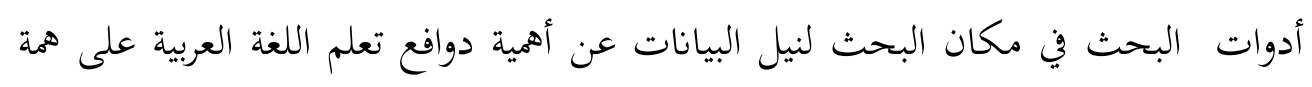

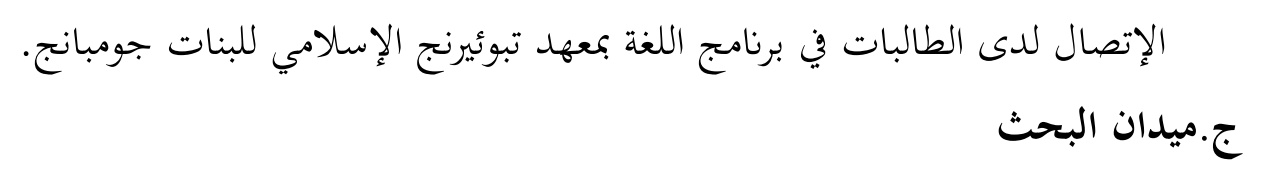

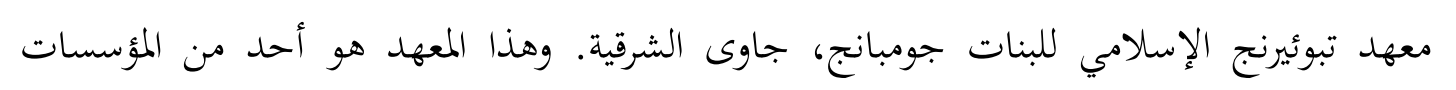

$$
\text { 1. الرممية هاشم أشعري ولديه البرامج التالية: }
$$

2. البرامج الخاصة تتكون من: تحفيظ القرآن، وكتاب التخصص من السلف، تعميق اللغات الأجنبية

$$
\text { (العربية والإنجليزية) }
$$


و يجب ان استخدام لغتان على الطالبات الذين يدخلون في برنامج اللغة في أنشطتهم

$$
\text { اليومية. }
$$

د. البيانات ومصادرها

أما هذا المصدر من الإخصاء المدقق الواقع المبحوث في الكتب والورقة المتعلق بالواقع والتاريخ والمقال.

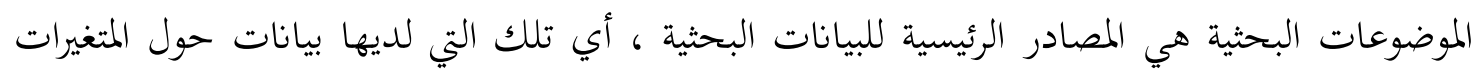

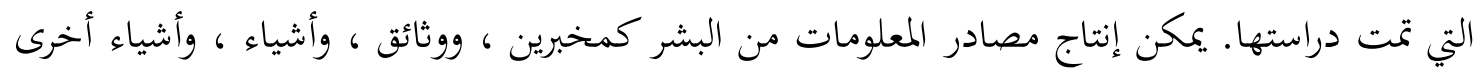

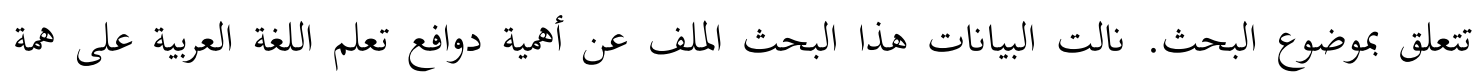
الإتصال . بها.

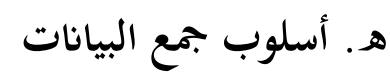

قد عمل جمع البيانات بأنواع الإطار والمنابع و الطروق. من جهة أسلوب جمع البيانات

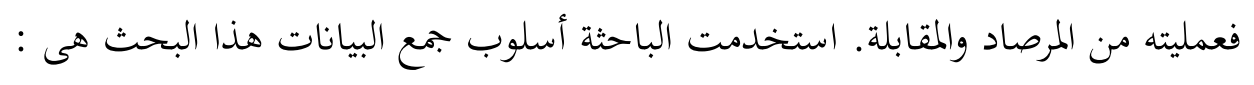

1. الملاحظة

الملاحظة من اللغة الاتينية بمعنى النظر و الإهتمام. يتوجه هذا الإصطلاح فن عملية الإهتمام الدقيق والكتابة الظاهرة النشأة والمتوزنة بين علقة الظاهرة.

عادة ما يتم تفسير طريقة المراقبة كطريقة علمية كملاحظة وتسجيل منهجي للظاهرة التي تم

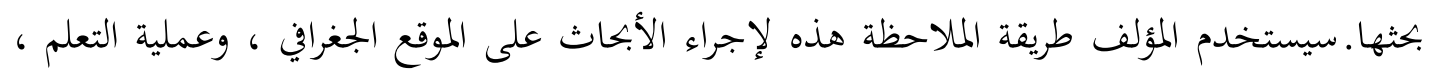

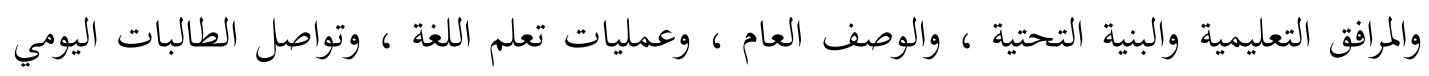

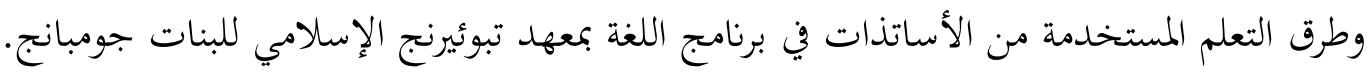
2. المقابلة

استخدمت المقابلة لأسلوب جمع البيانات حينما الباحثة تريد عملية البحث المقادم لتجيد

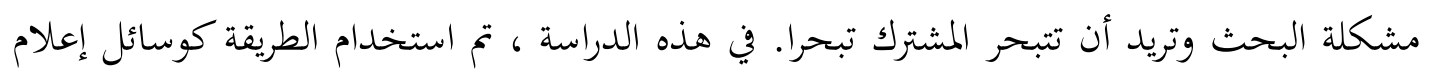
رئيسية للحصول على بيانات أولية من الأساتذات في برنامج اللغة وجزء من الطالبات. 
استخدم طريقة التوثيق هذه أيضًا لتحديد الشروط الموضوعية في في برنامج اللغة بمعهد تبوئيرنج

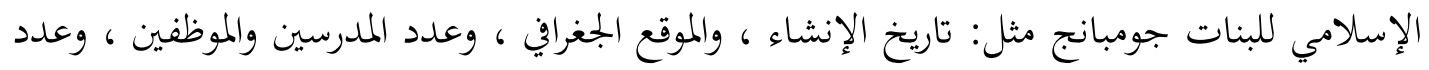
الطلاب ، والمرافق ، والهيكل التنظيمي. نتائج البحث من نتائج المقابلات السابقة أن يعرف على المنخفض دوافع التعلم اللغة العربية من بينها يمكن تفسيرها :

$$
\text { في حفظ المفردات، تكون الطالبات أبطأ من حفظ عocabularies }
$$

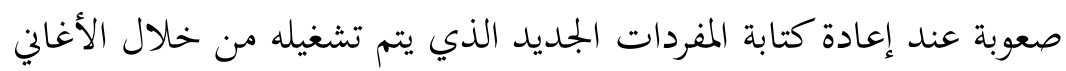

$$
\text { متحمس لتعلم اللغة العربية الضعيفة، عندما تكون الواجبات }
$$

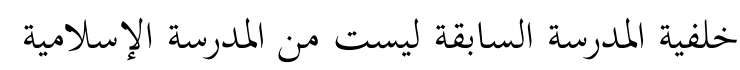

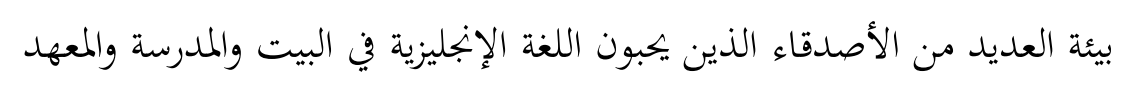

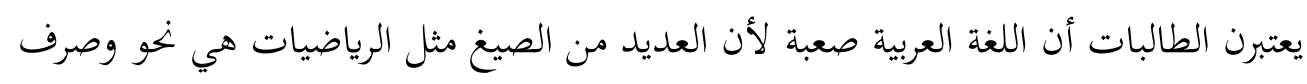
$-6$

$$
\begin{aligned}
& \text { خلفية الطالبات في دورة اللغة الإنجليزية } \\
& \text { من الصعب أن يفهم اللغة العربية } \\
& \text { غالبا ما ينسى في تقليد المفردات الجديدة } \\
& \text { غير واثق بتعلم اللغة العربية } \\
& \text { من الصعب جعل الجمل العربية } \\
& \text { أفن يفضلن سماع الموسيقى الإنجليزية من العربية الصعبة الحبية }
\end{aligned}
$$

من الشرح أعلاه تشير إلى أن الدوافع تعلم اللغة العربية تنتمي إلى المنخفضية من العرية، مع الخغناضه

$$
\begin{aligned}
& \text { للتواصل باللغة العربية لديهن صعوبات، بين تصريحاهن وهي: }
\end{aligned}
$$

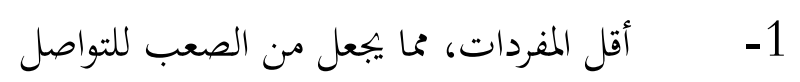

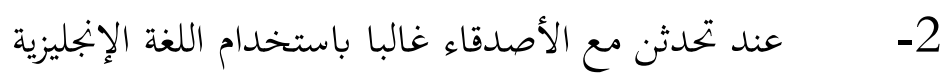

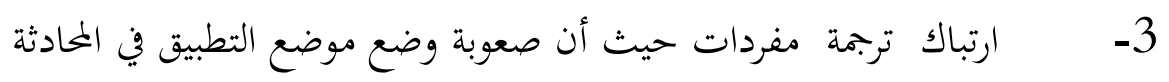

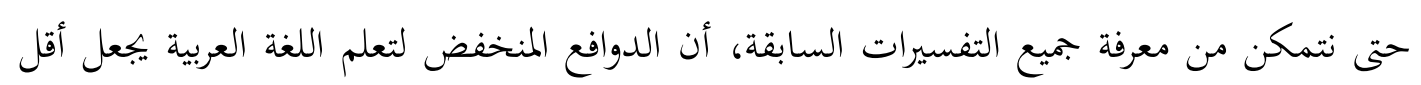

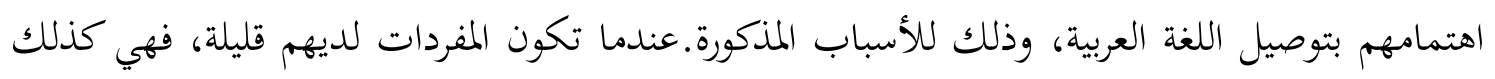


صعبة لتحدث باللغة العربية. لكذالك، دوافع تعلم باللغة العربية مهمة جدا لزيادة الاهتمام بالتواصل اللغة العربية.

الخايتمة

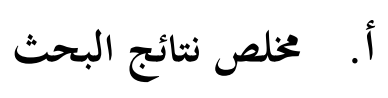

وأهم النتائج التي اظهرت الباحثة في برنامج اللغة بععهد تبوئيرنج الإسلامي للبنات جومبانج هذا البحث فهي

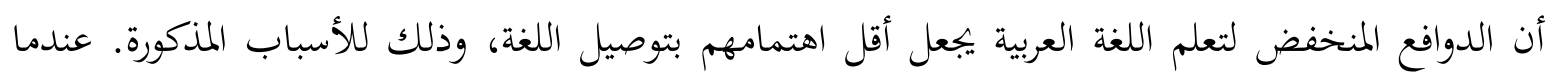

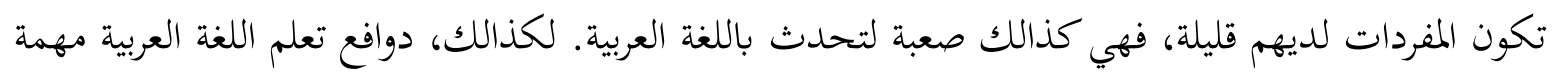
جدا لزيادة الإهتمام بالتواصل اللغة العربية.

\section{المراجع}

الفوزان،عبد الرمن إبراهيم، إضاءات لمعلمي اللغة العربية لغير الناطقين بما، الرياض: العربية للجميع، 2016 العبودي، فاطمة بنت محمد، استراتيجيات التعلم والتعليم والتقويم، مشروع التأسيس للجودة والتأهل للاعتماد المؤسسي والبرابجي.

عبدالكريم، طه علي حسين الدليمي وسعاد عبدالكريم، اللغة العربية مناهجها وطرائق تدريسها، البغداد: دارالشروق 2005

أمال، بن يوسف، العلاقة بين استراتيجيات التعلم والدافعية للتعلم وأثثرما على التحصيل الدراسي دراسة ميدانية على تلاميذ بعض الثانويات بولاية البليدة منكرة لنيل شهادة الماجسنير في علوم التربية، الجزائر: جامعة الجزائر كلية العلوم الإنسانية و الاجتماعية قسم علم النفس و علوم التربية و الأرطفونيا،2008 الهدى، مفتاح، العببية لأغراض خاصة (برنامج الخاص لتعليم اللغة العربية جامعة مولانا مالك إبراهيم الإسلامية الحكومية مالانق)

David Muljadi, Kamus Besar Bahasa Indonesia V 0.21

Rohman, Fathur , Metodologi Pembelajaran Bahasa Arab (Malang: Madani:2015) 2009)

Wahyuni,Esa Nur, Motivasi dalam Pembelajaran, (Malang:Uin-Malang Press,

Mulyana, Dedy ,Ilmu Komunikasi, , (Bandung: Remaja Rosdakarya, 2009) 


$$
\text { أهمية دوافع تعلم اللغة العربية لدى الطالبات على همة الإتصال بما, جميعة الخيرية }
$$

Arifin, Zainal, PenelitianPendidikanMetodedanParadigmaBaru, (Bandung : PT RemajaRosdakarya, 2012

Moleong, Lexy J., Metodologi Penelitian Kualitatif, (Bandung: PT. Remaja Rosdakarya, 2015

Tohirin, Metodologi Penelitian Kualitatif dalam Pendidikan dan Bimbingan Konseling (Jakarta : Raja Grafindo Persada, 2012) 2014)

Ahmadi,,Rulam, Metodologi Penelitian Kualitatif (Yogyakarta: Ar-Ruzz Media,

Sukardi, Metodologi penelitian Pendidikan: Kompetensidan Praktiknya, (Jakarta : Bumi Aksara, 2003)

Azwar, Saifuddin, Metode Penelitian, cet. II, (Yogyakarta: Pustaka Pelajar, 1999)

A.Maolani, Rukaesih, MetodologiPenelitianPendidikan, (Jakarta : PT. Raja GrafindoPersada, 2015

Unawan,Imam, Metodepenelitiankualitatifteoridanpraktik, (Jakarta : PT. BumiAksara, 2013

Hadi, Sutrisno, Metode Researh Jilid II, (Yogyakarta : Andi Offset, 2001)

Tanzeh, Ahmad, Pengantar Metode Penelitian, (Yogyakarta: Teras, 2009),

http://duniakampus7.blogspot.com/2014/03/pengaruh-minat-belajar-bahasaarab 2028.html

https://saaid.net/aldawah/238.htm 